\title{
In-utero treatment of bilateral renal agenesis: a threshold analysis of possible cost effectiveness
}

\author{
Abstract \\ Objective: Recently amnioinfusion in a case of fetal bilateral renal agenesis resulted in a \\ live-born neonate with gestational age-appropriate lung function. While this intervention is \\ novel, its long term effects are uncertain. We sought to determine under what conditions this \\ intervention would be cost effective. \\ Methods: Decision-analysis modeling was used to compare serial amnioinfusion to routine \\ care following diagnosis of bilateral renal agenesis in singleton gestations with normal \\ karyotype. Assumptions included \\ i. serial amnioinfusions allow for improved respiratory function at birth, \\ ii. peritoneal dialysis was started in the newborn period. \\ iii. If the neonate survived dialysis, he/she underwent transplant at 2 years of age.
}

Volume 2 Issue 3 - 2015

\author{
Erika FWerner,' Alisse Hauspurg,' Jessica L \\ Bienstock ${ }^{2}$ \\ 'Women \& Infants Hospital, Alpert Medical School at Brown \\ University, USA \\ ${ }^{2}$ Gynecology and Obstetrics, Johns Hopkins University Medical \\ School, USA
}

\begin{abstract}
Correspondence: Erika FWerner, 101 Dudley St, Providence, RI 02906, USA, Tel 40I-274-I I 22, Fax 40 I-453-7622,
\end{abstract} Email ewerner@wihri.org

Received: February 12, 2015 | Published: April 22, 2015

Costs included amnioinfusions, delivery, NICU care, dialysis and transplant. The main outcome measure was incremental cost-effectiveness ratio (ICER) defined as marginal cost per quality-adjusted life year (QALY) gained. An ICER less than \$100,000/QALY gained were considered cost effective. Sensitivity analyses were performed to assess thresholds at which serial amnioinfusions were cost effective.

Results: Our model predicts that there are plausible conditions under which serial amnioinfusion is cost effective for bilateral renal agenesis. Threshold analysis revealed that serial amnioinfusion must achieve gestational-age appropriate lung function in $12 \%$ of cases for intervention to be cost-effective. Time on dialysis, probability of survival to transplant, overall life expectancy and quality of life after transplant significantly affect whether this intervention is cost effective.

Conclusion: A prospective trial investigating the effect of serial amnioinfusion in fetuses with bilateral renal agenesis is needed that evaluates the variables to which this model was most sensitive.

Keywords: fetal renal agenesis, potters syndrome, cost-effective analysis, amnioinfusion, decision model

Abbreviations: BRA, bilateral renal agenesis; PD, peritoneal dialysis; QAYLs, quality adjusted life years; RR, relative risk; ICER, incremental cost-effectiveness ratio

\section{Introduction}

Bilateral renal agenesis (BRA) is a severe fetal anomaly that occurs in 1 in 3000 pregnancies. $^{1}$ This diagnosis was previously considered uniformly fatal with fetuses either dying in-utero from cord compression or newborns dying within hours of birth due to severe pulmonary hypoplasia. Recently Bienstock et al. ${ }^{2}$ reported on a singleton with BRA who received serial amnioinfusions starting at 24 weeks. This pregnancy was complicated by preterm labor. The neonate was delivered at almost 29 weeks and was found at delivery to have age appropriate lung function. The child is currently 1 year old and on peritoneal dialysis. ${ }^{2}$ Previously, women who were found to have a singleton with BRA had no options available to alter the prognosis for their neonate. This case report represents a possible drastic change in how pregnancies complicated by BRA will be counseled. Obviously, no one case report should change practice. However, as we wait for prospective cohort studies evaluating the effects of serial amnioinfusion on outcomes of neonates with BRA, we must consider how this intervention may affect long-term health and care costs. Even if intrauterine death from cord compression and neonatal pulmonary hypoplasia can be prevented with serial amnioinfusions, newborns with BRA face a lifetime of medical interventions. Without kidneys or the maternal-placental interface to dispose of metabolic waste products, dialysis is required until transplant can be achieved. While peritoneal dialysis (PD) for infants has recently become available yielding survival rates as high at $89 \%$ at 12 months, these children will eventually need renal transplants. ${ }^{3}$ Even post-transplant, survivors face significant morbidity and likely a shortened life expectancy. As pointed out in the accompanying editorial to the Bienstock et al. ${ }^{2}$ report, "even if sound clinical research can determine the efficacy of a fetal intervention, success no longer can be defined as neonatal survival or short-term outcome. Long-term survival and quality of life in infancy and childhood must be taken into account". ${ }^{4}$ Given the numerous costs and effects associated with all of these interventions, we developed a decision model to determine under what thresholds serial amnioinfusion for BRA would be costeffective from a health care perspective. 


\section{Methods}

A decision analytical model was designed using TreeAge Pro 2011 (TreeAge Software, Williamstown, MA) that compared attempted intervention for renal agenesis with serial amnioinfusions versus routine care. In the intervention group, the following assumptions were made,

a. Amnioinfusions were started at 20-24 weeks and continued weekly until delivery with the goal of improved neonatal lung function;

b. 48 hours after delivery, neonatal peritoneal dialysis was initiated;

c. In surviving children, renal transplant occurred at 2 years of life. In the routine care group, the renal agenesis was assumed to be lethal for the neonate and only comfort care was provided.
The base case probability estimates used in the decision model were chosen from data in the published literature whenever possible. To obtain these estimations, a bibliographic survey in PUBMED was performed using the following search terms: renal agenesis, neonatal dialysis, renal transplant, preterm birth, Potters syndrome, cost, utility, life expectancy and combinations of these terms. Additionally, the bibliographies of the articles identified during these searches were used as reference sources. As there are no randomized controlled trials evaluating renal agenesis, dialysis or renal transplant in children, point estimates were determined from prospective cohorts when possible. The probability and utility estimates used in support of our model are reported in Table 1. Given the ambiguity of some of these data points, wide ranges were used in sensitivity analysis to assess thresholds at which strategies shifted from not cost effective to cost effective and to cost saving.

Table I Estimates of probabilities, utilities and life expectancies used in the model

\begin{tabular}{|c|c|c|c|c|}
\hline Probabilities & Base case & Minimum & Maximum & References \\
\hline Likelihood of delivery at & & & & $5,6,20$ \\
\hline I. $>24$ weeks to $<28$ weeks & $6.20 \%$ & $0.00 \%$ & $50 \%$ & \\
\hline I. $>28$ weeks to $<34$ weeks & $17.70 \%$ & $0.00 \%$ & $50 \%$ & \\
\hline I. $>34$ weeks & $76.10 \%$ & $0.00 \%$ & $100 \%$ & \\
\hline Neonatal survivability with serial amnioinfusion & & & & Internal data, ${ }^{8}$ \\
\hline I. $>24$ weeks to $<28$ weeks & $41.10 \%$ & $0.00 \%$ & $82.20 \%$ & \\
\hline I. $>28$ weeks to $<34$ weeks & $49.50 \%$ & $0.00 \%$ & $99.30 \%$ & \\
\hline I. $>34$ weeks & $49.90 \%$ & $0.00 \%$ & $99.30 \%$ & \\
\hline Time to renal transplant & 2 years & I year & 10 years & 12 \\
\hline Surviving to renal transplant & $63.30 \%$ & $0.00 \%$ & $90.00 \%$ & 3,9-12 \\
\hline Surviving first year post-transplant & $91.70 \%$ & $80 \%$ & $100 \%$ & 11,12 \\
\hline Likelihood of requiring a second renal transplant & $17 \%$ & $1.80 \%$ & $23.00 \%$ & 11,12 \\
\hline Life expectancy if alive one year after transplant & 19 years & 10 years & 76 years & 11 \\
\hline Utilities for & & & & 13 \\
\hline I. Dialysis & 0.72 & 0.65 & 0.75 & \\
\hline I.Transplant & 0.8 & 0.69 & 0.9 & \\
\hline I. Death & 0 & 0 & 0 & \\
\hline Discounted rate for utilities based on delivery at & & & & 8 \\
\hline I. $>24$ weeks to $<28$ weeks & 0.95 & - & 1 & 14 \\
\hline I. $>28$ weeks to $<34$ weeks & 0.98 & - & I & 13 \\
\hline I. $>34$ weeks & 0.99 & - & 1 & 15 \\
\hline
\end{tabular}

Cost effectiveness and cost saving were determined by the incremental cost-effectiveness ratio (ICER). ICER measures the amount of additional expense required to obtain one perfect year of health. Health is measured in quality adjusted life years (QAYLs) which is a sum of health each year over the course of a life time (utility $\mathrm{x}$ life expectancy). For this study, serial amnio-infusion compared to routine care was judged to be cost effective if the ICER was less than $\$ 100,000$ per QALY gained. An ICER of $\$ 100,000$ is the threshold typically cited in the medical literature as the amount society is willing to pay to gain a perfect year of health. In our sensitivity analysis and our Monte Carlo simulation, we allowed society willingness to pay for each QALY gained to range from $\$ 0$ to $\$ 200,000$.

\section{Probabilities}

Table 1 highlights that neonates in the intervention group were assumed to deliver prematurely as was the case in the single neonate 
who received serial amnioinfusion for renal agenesis. We based the estimates on the premise that $0.59 \%$ of uncomplicated pregnancies deliver between 24 and 28 weeks and an additional $2.18 \%$ deliver between 28 and 34 weeks. ${ }^{5}$ These rates were then multiplied by the relative risk (RR) of very preterm ( 24 to 28 weeks), preterm (28 to 34 weeks) and late preterm (34 to 36 weeks) deliveries for pregnancies complicated by renal agenesis based on population-based cohorts ${ }^{6,7}$ and by the relative risk of preterm delivery due to repeated amniocenteses. In our base case, we assumed no increased risk of very preterm or preterm delivery due to serial amnioinfusion. However, as there is a theoretical risk that repeats amniocentesis due to serial amnioinfusions may significantly increase the risk of preterm labor and infection, we allowed the RR of preterm birth to increase by a factor of 4.1 in the sensitivity analysis.

To obtain the likelihood of survival at birth, we assumed survival in an otherwise healthy neonate was dependent of the gestational age at birth; $82.1 \%$ at $24-28$ weeks, $99.1 \%$ at $28-34$ weeks and $99.8 \%$ at greater than 34 weeks [8]. For the neonates in this model, the probability of survival based on gestational age at delivery was then multiplied by a relative risk of survival given the BRA. For the group without intervention, this relative risk was assumed to be zero as BRA is a lethal condition. With serial amnioinfusion, this relative risk was assumed to be 0.5 in the base case; $50 \%$ of BRA pregnancies receiving serial amnioinfusion would result in sufficient lung volumes to survive. Given that as of yet, there is only one case report suggesting efficacy of serial amnioinfusion, we chose a RR of 0.5 in the base case but allowed this RR to vary from 0 to 1 in the threshold analysis.

As expected there is no published literature on dialysis or renal transplant in infants with renal agenesis. Thus we obtained estimates for survival on dialysis, time to transplant, survival post-transplant and costs relating to dialysis and renal transplant from cohort studies performed in neonates with other conditions, such as posterior urethral valves, or multicystic dysplastic kidneys. ${ }^{3,9-12}$ Given the uncertainty of how renal agenesis infants will do with dialysis even if their lung function is optimized, we used an extremely wide interval in the threshold analysis.

Table 2 Costs relating to serial amnioinfusion for renal agenesis

\begin{tabular}{|c|c|c|c|c|}
\hline Costs in US 2013 dollars & Base case & Minimum & Maximum & References \\
\hline \multicolumn{4}{|l|}{ Delivery with intervention } & \multirow[t]{4}{*}{15,22} \\
\hline I.Very preterm & 234,202 & 97,079 & 468,477 & \\
\hline I. Preterm & 48,484 & 20,664 & 96,981 & \\
\hline I. Late preterm & 16,343 & 11,324 & 23,095 & \\
\hline Delivery with expectant management & 3,827 & 1,914 & 7,656 & 15,22 \\
\hline Amnioinfusion (per week) & 1,000 & 500 & 3,000 & 20 \\
\hline Dialysis per year & 69,592 & 38,819 & 124,998 & $16-18$ \\
\hline Renal Transplant ${ }^{*}$ & 58,403 & 10,000 & 150,000 & 19,20 \\
\hline Immunosuppression per year & 12,225 & 1,000 & 50,000 & 19 \\
\hline
\end{tabular}

*Includes pre-op evaluation and first year post-transplant care.

For a cohort of 100 pregnant women carrying singleton fetuses with renal agenesis and no other anomalies (normal karyotypes), we calculated the ICER of serial amnioinfusion versus routine care. We performed threshold analyses by varying the values of the variables in the model to their plausible extremes. We also allowed all variables

\section{Utilities}

Based upon the available literature, utilities were given to the children on dialysis and status post renal transplant (Table 1). Utilities are a means to evaluate the relative quality of life as compared to health. We determined three health states that would be relevant to infants born with renal agenesis in our analysis: death (utility 0 ), dialysis (utility 0.72 ), status post transplant (utility 0.8 ). ${ }^{13}$ These utilities were then further discounted based on the risk of severe neurologic injury associated with very preterm, preterm and late preterm delivery. ${ }^{8,13-15}$ We assumed that only $63.3 \%$ of the neonates who survived the neonatal period lived to 2 years of age when they were large enough to undergo renal transplant. ${ }^{12}$ Additionally the model assumed slightly more than $9 \%$ of the children receiving transplant died in the first year after transplant and that an additional $16.7 \%$ (range 1.8-23\%) had to return to dialysis during their life and undergo a second renal transplant. ${ }^{3,9-12}$ The life expectancy of children who receive renal transplants is very uncertain. We estimated a median life expectancy of 19 years for children with renal agenesis who successfully survived to the first year after renal transplant. Again we used a wide range for life expectancy when calculating the quality adjusted life years (QALYs) given the lack of data available. ${ }^{11}$ QALYs were calculated by obtaining the sum of all yearly utilities over the life expectancy. Future utilities were discounted at a baseline rate of $3 \%$ per year.

\section{Costs}

Cost data were derived from the published literature (Table 2). ${ }^{16-22}$ All costs are presented in 2013 United States dollars and are adjusted based on the use of the medical care component of the Consumer Price Index. In the serial amnioinfusion strategy, delivery was assumed to involve full neonatal intervention. While in the routine care strategy, delivery was assumed to involve delivery and comfort care. Dialysis was assumed to be peritoneal as this is the most common type of dialysis with infants. Like utilities, costs were discounted at a baseline rate of 3\% based on average inflation. The range was varied from $1-5 \%$ in the sensitivity analysis. All analyses were from a health care system perspective. to fluctuate simultaneously with 10,000 trials in a Monte-Carlo simulation. Other parameters we estimated include: total cost of each strategy, total QALYs per strategy and percentage of treated neonates with adequate lung function to make amnioinfusion cost-effective. 


\section{Results}

Our analysis suggests that under the base case probabilities and costs presented in Table $1 \&$ Table 2, assuming 50\% of neonates have adequate pulmonary function with serial amnioinfusion, intervention for BRA is cost effective from a health care perspective with an ICER of $\$ 54,961$ per QALY gained. For a theoretical cohort of 100 cases of BRA, serial amnioinfusion would cost the health system an additional $\$ 18,436,083$ and lead to 31 neonates surviving to 2 years of life and 29 children surviving to 1 year post-transplant resulting in 335 more QALYs compared to routine care.

As the data points inputted into the model are relatively uncertain, a threshold analysis was performed to identify critical values that would make serial amnioinfusion cost-effective or not cost-effective. The model was most sensitive to the probability that amnioinfusion would lead to lung function compatible with life. When less than $12 \%$ of treated neonates have the necessary lung function for survival, serial amnioinfusion is no longer cost-effective, costing more than $\$ 100,000$ per QALY. The model was also sensitive to the number of years that dialysis was required prior to transplant, the probability of surviving on dialysis until transplant, and the utility and life expectancy after transplant (Figure 1). If dialysis was required for more than 9 years after birth, serial amnioinfusions ceased to be costeffective. Additionally if less than $15 \%$ of the neonates who start dialysis live to the age of two to receive transplant, treatment is not cost-effective. Finally, if the QALYs after transplant at age 2 are less than 5.9 either due to the utility, the life expectancy or the discounting rate, then serial amnioinfusion during pregnancy is not cost effective.

Monte Carlo simulation, a computational algorithm that relies on repeated random sampling, was also used to simultaneously vary all variables across the extreme ranges listed in Tables $1-3$. With 10,000 simulations, serial amnioinfusion was cost-effective $76.2 \%$ of the time. As expected, it was never cost-saving. Interestingly, if the willingness to pay was shifted to $\$ 50,000$ from $\$ 100,000$ per QALY gained, serial amnioinfusion is only cost-effective $10 \%$ of the time (Figure 2).

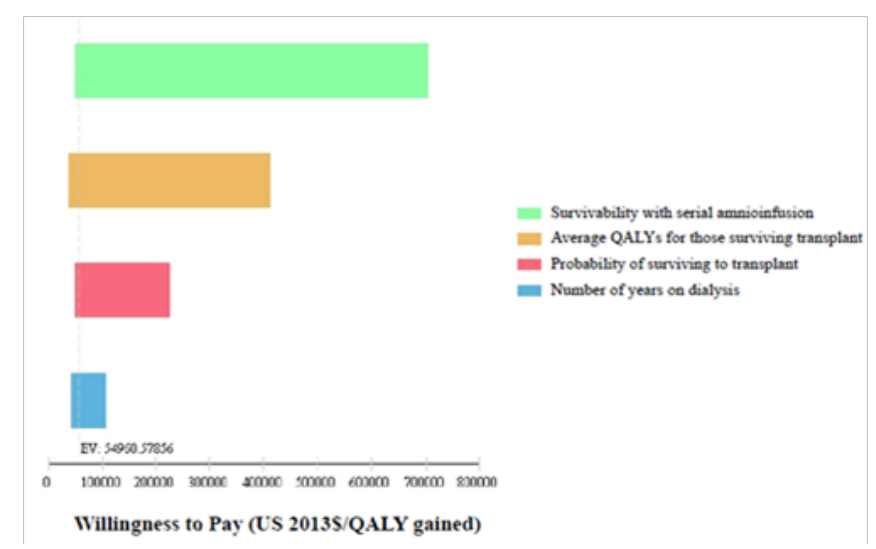

Figure I Tornado Diagram of critical values in one way sensitivity

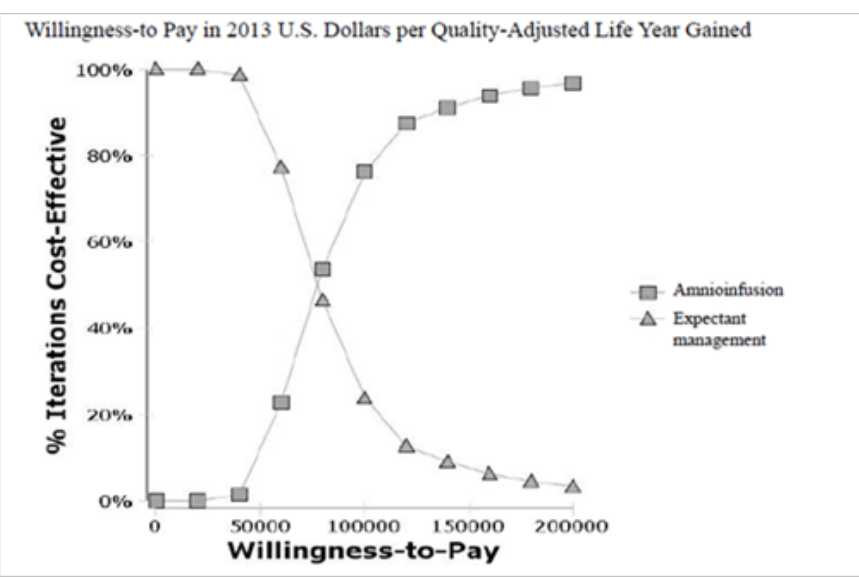

analysis.Figure 2 Cost-effective acceptability curve.

Table 3 Summary of results (per 100 cases)

\begin{tabular}{llll}
\hline Variable & Routine care & Serial amnioinfusion & Marginal difference \\
\hline Number of amnioninfusion & 0 & 1280 & 1280 \\
Survival to transplant & 0 & 31 & 31 \\
Survival to one year post transplant & 0 & 29 & 29 \\
Need for retransplant & 0 & 5 & 5 \\
Cost & $\$ 382,700$ & $\$ 18,818,783$ & $\$ 18,436,083$ \\
QALYs for child & 0 & 335.44 & 335.44 \\
Marginal cost/QALY gained & - & - & $\$ 54,961$ \\
\hline
\end{tabular}

*All costs are in 2013 U.S. dollars.

\section{Discussion}

As fetal therapy moves forward, we are likely to see more interventions that offer hope in conditions previously considered lethal. However, as with all new therapies, care must be taken to consider not just the short-term ramifications of the intervention but how both maternal and child health will be affected over the life course. This model suggests that serial amnioinfusion in cases of fetal bilateral renal agenesis may be a cost-effective intervention under plausible conditions and therefore warrants further prospective studies. This model also suggests that serial amnioinfusion for bilateral renal agenesis may not be cost effective if prospective studies demonstrate only a small benefit. Our study highlights the outcomes that a prospective study must evaluate if we are to determine whether this intervention is cost-effective. These include not only the rate of neonatal survival following serial amnioinfusion but also the rate of survival to transplant, the age at transplant and the quality of life and longevity following transplant.

Given the absence of data on the long-term outcomes of neonates who receive serial amnioinfusions for BRA, we fully acknowledge that this cost-effective model has significant limitations. Even if we 
accept the assumption that serial amnioinfusions are successful at reducing stillbirth rates and neonatal death rates and allow for normal fetal lung development, we have no first hand data on how these children will respond long term to dialysis or renal transplant. For this study, we extrapolated from other renal disorders that begin in utero, but it is possible that the difference in etiology will lead to a significantly different response to the same interventions. Thus the ICERs calculated should not be taken as an exact measure but as an indicator that despite the years of dialysis, eventual transplant and post-transplant care are required for these children, there are plausible conditions in which intervention could be cost-effective from a health care perspective.

In fact, this model is intended not to demonstrate that amnioinfusion is or is not cost effective but instead to identify which data points most greatly impact the cost and effect analysis. Perhaps future research can be focused on obtaining that information. Too often obstetrical studies focus only on the immediate outcome of surviving through the neonatal period. Even if we achieve lung function in BRA-affected neonates, serial amnioinfusions for BRA pose a risk of infection to the mother and the risk of false hope to the family. Furthermore, BRA may still be a lethal condition prior to reaching childhood. This decision model highlights that efficacy of serial amnioinfusion cannot be judged by survival into infancy but must also be assessed in terms of survival to transplant and quality of life and longevity post-transplant.

As we wait for prospective long-term data, it seems reasonable to discuss serial amnioinfusions with patients who desire to continue pregnancy when BRA is diagnosed. However, like other novel fetal interventions, interested patients must be made aware that the outcomes remain uncertain. Ideally, women who move forward with this intervention will be part of a study protocol so that over time, we will have short and long term data to offer patients regarding survival rates following neonatal dialysis and transplant, incidence and prevention of organ rejection and quality of life issues post-transplant.

\section{Acknowledgments}

None.

\section{Conflicts of interest}

The authors declare there is no conflict of interests.

\section{References}

1. Woodward PJ, Kennedy A, Sohaey R, et al. Diagnostic imaging: obstetrics. Amirsys, Salt Lake City, UT 84106, USA; 2011:2-28.

2. Bienstock JL, Birsner ML, Coleman F, et al. Successful in utero intervention for bilateral renal agenesis. Obstet Gynecol. 2014;124(2P 2 Suppl 1):413-415.

3. Vidal E, Edefonti A, Murer L, et al. Peritoneal dialysis in infants: the experience of the Italian Registry of Paediatric Chronic Dialysis. Nephrol Dial Transplant. 2012;27(1):388-395.

4. Johnson A, Luks FI. A Cautionary Note on New Fetal Interventions. Obstet Gynecol. 2014;124(2 Pt 2 Suppl 1):411-412.
5. Martin JA, Hamilton BE, Ventura SJ, et al. Births: final data for 2011. National vital statistics reports, National Center for Health Statistics, Hyattsville, MD, USA, 2013;62(1):1-70.

6. Purisch SE, DeFranco EA, Muglia LJ, et al. Preterm birth in pregnancies complicated by major congenital malformations: a population-based study. Am J Obstet Gynecol. 2008;199(3):287e1-287e8.

7. Locatelli A, Vergani P, DiPirro G, et al. Role of amnioinfusion in the management of premature rupture of the membranes at $<26$ weeks gestation. Am J Obstet Gynecol. 2000;183(4):878-882.

8. Clements KM, Barfield WD, Ayadi MF, et al. Preterm birth-associated cost of early intervention services: an analysis by gestational age. Pediatrics. 2007;119(4):e866-e874.

9. Carey WA, Talley LI, Sehring SA, et al. Outcomes of dialysis initiated during the neonatal period for treatment of end-stage renal disease: A North American Pediatric Renal Trials and Collaborative Studies special analysis. Pediatrics. 2007;119(2):e468-e473.

10. Mitsnefes MM, Laskin BL, Dahhou M, et al. Mortality risk among children initially treated with dialysis for end-stage kidney disease, 19902010. JAMA. 2013;309(18):1921-1929.

11. Hijazi R, Abitbol CL, Chandar J, et al. Twenty-five years of infant dialysis: a single center experience. J Pediatr. 2009;155(1):111-117.

12. Alexander RT, Foster BJ, Tonelli MA, et al. Survival and transplantation outcomes of children less than 2 years of age with end-stage renal disease. Pediatr Nephrol. 2012;27(10):1975-1983.

13. Tengs TO, Wallace A. One thousand health-related quality-of-life estimates. Med Care. 2000;38(6):583-637.

14. Moster D, Lie RT, Markestad T. Long-term medical and social consequences of preterm birth. N Engl J Med. 2008;359(3):262-273.

15. Werner EF, Han CS, Pettker CM, et al. Universal cervical-length screening to prevent preterm birth: a cost-effectiveness analysis. Ultrasound Obstet Gynecol. 2011;38(1):32-37.

16. Coyte PC, Young LG, Tipper BL, et al. An economic evaluation of hospital-based hemodialysis and home-based peritoneal dialysis for pediatric patients. Am J Kidney Dis. 1996;27(4):557-565.

17. Berger A, Edelsberg J, Inglese GW, et al. Cost comparison of peritoneal dialysis versus hemodialysis in end-stage renal disease. Am J Manag Care. 2009;15(8):509-518.

18. Bruns FJ, Seddon P, Saul M, et al. The cost of caring for end-stage kidney disease patients: an analysis based on hospital financial transaction records. J Am Soc Nephrol. 1998;9(5):884-890.

19. Kerr M, Bray B, Medcalf J, et al. Estimating the financial cost of chronic kidney disease to the NHS in England. Nephrol Dial Transplant. 2012;27 Suppl 3:iii73-iii80.

20. Ohno M, Caughey A. The role of noninvasive prenatal testing as a diagnostic versus a screening tool--a cost-effectiveness analysis. Prenat Diagn. 2013;33(7):630-635.

21. Whiting JF, Woodward RS, Zavala EY, et al. Economic cost of expanded criteria donors in cadaveric renal transplantation: analysis of Medicare payments. Transplantation. 2000;70(5):755-760.

22. Gilbert WM, Nesbitt TS, Danielsen B. The cost of prematurity: quantification by gestational age and birth weight. Obstet Gynecol. 2003;102(3):488-492. 